\title{
A Lanthanide Phosphinidene Complex: Synthesis, Structure, and Phospha-Wittig Reactivity
}

\author{
Jason D. Masuda,${ }^{\dagger}$ Kimberly C. Jantunen, ${ }^{\dagger}$ Oleg V. Ozerov, ${ }^{\star}$ Kevin J. T. Noonan,${ }^{\S}$ \\ Derek P. Gates, ${ }^{\S}$ Brian L. Scott ${ }^{\dagger}$ and Jaqueline L. Kiplinger* ${ }^{\dagger}$ \\ ${ }^{\dagger}$ Los Alamos National Laboratory, Mail Stop J514, Los Alamos, New Mexico, 87545, USA; \\ ${ }^{\ddagger}$ Department of Chemistry, Brandeis University, MS015, 415 South Street, Waltham, MA, 02454, \\ USA; ${ }^{\S}$ Department of Chemistry, University of British Columbia, 2036 Main Mall, Vancouver, \\ British Columbia, V6T 1Z1, Canada
}

\section{Supporting Information}

- General Synthetic Considerations. $\quad$ S2

- Synthesis of $\mathrm{Lu}\left(\mathrm{CH}_{2} \mathrm{SiMe}_{3}\right)_{3}(\mathrm{THF})_{2}$. $\quad$ S3

- Synthesis of $\mathrm{MesPH}_{2}$. S3

- Synthesis of $\left[\left\{2-\left(\mathrm{Ph}_{2} \mathrm{P}\right)-4-\mathrm{Me}-\mathrm{C}_{6} \mathrm{H}_{3}\right\}_{2} \mathrm{~N}\right] \mathrm{H}(2) . \quad \mathrm{S4}$

- Synthesis of $\left[\left\{2-\left({ }^{i} \mathrm{Pr}_{2} \mathrm{P}\right)-4-\mathrm{Me}-\mathrm{C}_{6} \mathrm{H}_{3}\right\}_{2} \mathrm{~N}\right] \mathrm{Lu}\left(\mathrm{CH}_{2} \mathrm{SiMe}_{3}\right)_{2}(3)$.

- Synthesis of $\left[\left\{2-\left(\mathrm{Ph}_{2} \mathrm{P}\right)-4-\mathrm{Me}_{-} \mathrm{C}_{6} \mathrm{H}_{3}\right\}_{2} \mathrm{~N}\right] \mathrm{Lu}\left(\mathrm{CH}_{2} \mathrm{SiMe}_{3}\right)_{2}(4) . \quad$ S5

- Synthesis of $\left[\left\{2-\left({ }^{i} \mathrm{Pr}_{2} \mathrm{P}\right)-4-\mathrm{Me}-\mathrm{C}_{6} \mathrm{H}_{3}\right\}_{2} \mathrm{NLu}\right]_{2}(\mu \text {-PMes) })_{2}(\mathbf{5}) . \quad$ S6

- Procedure for Generation of $E-\mathrm{Mes}=\mathrm{C}(\mathrm{H})^{t} \mathrm{Bu}$. S7

- Procedure for Generation of $\mathrm{Mes}=\mathrm{CPh}_{2}$. S7

- Generation of Phosphaindole 6. S7

- Crystallographic Details for Complexes 3 and $5 . \quad$ S9

- Table S1. Crystal Data and Structure Refinement for [2-( $\left.\operatorname{Pr}_{2} \mathrm{P}\right)-4-\mathrm{Me}-\quad$ S10 $\left.\left.\mathrm{C}_{6} \mathrm{H}_{3}\right\}_{2} \mathrm{~N}\right] \mathrm{Lu}\left(\mathrm{CH}_{2} \mathrm{SiMe}_{3}\right)_{2}(3)$.

- Table S2. Bond Lengths $[\AA]$ for $\left.\left[2-\left({ }^{i} \mathrm{Pr}_{2} \mathrm{P}\right)-4-\mathrm{Me}_{-} \mathrm{C}_{6} \mathrm{H}_{3}\right\}_{2} \mathrm{~N}\right] \mathrm{Lu}\left(\mathrm{CH}_{2} \mathrm{SiMe}_{3}\right)_{2}(3)$.

- Table S3. Bond Angles [ $\left.{ }^{\circ}\right]$ for $\left.\left[2-\left({ }^{i} \mathrm{Pr}_{2} \mathrm{P}\right)-4-\mathrm{Me}-\mathrm{C}_{6} \mathrm{H}_{3}\right\}_{2} \mathrm{~N}\right] \mathrm{Lu}\left(\mathrm{CH}_{2} \mathrm{SiMe}_{3}\right)_{2}(3) . \quad$ S12-S13

- Table S4. Crystal Data and Structure Refinement for $\left[\left\{2-\left({ }^{i} \operatorname{Pr}_{2} \mathrm{P}\right)-4-\mathrm{Me}-\mathrm{C}_{6} \mathrm{H}_{3}\right\}_{2} \mathrm{NLu}\right]_{2}(\mu-\quad \mathbf{S 1 4}$ PMes) $)_{2}(5)$.

- Table S5. Bond Lengths $[\AA]$ for $\left[\left\{2-\left({ }^{i} \mathrm{Pr}_{2} \mathrm{P}\right)-4-\mathrm{Me}-\mathrm{C}_{6} \mathrm{H}_{3}\right\}_{2} \mathrm{NLu}\right]_{2}(\mu-\mathrm{PMes})_{2}(5) . \quad$ S15-S16

- Table S6. Bond Angles $\left[{ }^{\circ}\right]$ for $\left[\left\{2-\left({ }^{i} \mathrm{Pr}_{2} \mathrm{P}\right)-4-\mathrm{Me}-\mathrm{C}_{6} \mathrm{H}_{3}\right\}_{2} \mathrm{NLu}\right]_{2}(\mu-\mathrm{PMes})_{2}(5) . \quad$ S17-S19

- References. $\quad$ S20 
General Synthetic Considerations. All reactions and manipulations were carried out using a Vacuum Atmospheres (MO 40-2 Dri-train) recirculating nitrogen atmosphere drybox, or using standard Schlenk and high vacuum line techniques. Glassware was dried at $150{ }^{\circ} \mathrm{C}$ before use. ${ }^{1} \mathrm{H},{ }^{13} \mathrm{C}\left\{{ }^{1} \mathrm{H}\right\}$, DEPT-90 and DEPT-135 spectra were collected using a Bruker Avance $300 \mathrm{MHz}$ spectrometer. ${ }^{1} \mathrm{H}$ NMR chemical shifts were referenced to the protio solvent impurity in benzene- $d_{6}$ at $\delta 7.16 \mathrm{ppm}$ or THF- $d_{8}$ at $\delta 3.58 \mathrm{ppm} .{ }^{13} \mathrm{C}\left\{{ }^{1} \mathrm{H}\right\}$ NMR chemical shifts were referenced to the solvent signal in benzene- $d_{6}$ at $\delta 128.39 \mathrm{ppm}$ or in THF- $d_{8}$ at $\delta 67.40 \mathrm{ppm}$. For ${ }^{31} \mathrm{P}$ NMR spectra, $\mathrm{H}_{3} \mathrm{PO}_{4}$ was used as an external reference at $\delta 0.00 \mathrm{ppm} .{ }^{13} \mathrm{C}\left\{{ }^{1} \mathrm{H}\right\} \mathrm{NMR}$ assignments were confirmed through the use of DEPT-90 and DEPT-135 NMR experiments.

Melting points were determined with a Mel-Temp II capillary melting point apparatus equipped with a Fluke $51 \mathrm{II} \mathrm{K/J}$ thermocouple using capillary tubes flame-sealed under nitrogen; values are uncorrected. Elemental Analyses were performed at the University of California, Berkeley Microanalytical Facility on a Perkin-Elmer Series II 2400 CHNS analyzer.

Unless otherwise noted, reagents were purchased from commercial suppliers and used without further purification. Celite (Aldrich), $4 \AA$ molecular sieves (Aldrich), and alumina (Brockman I, Aldrich) were dried under dynamic vacuum at $250{ }^{\circ} \mathrm{C}$ for $48 \mathrm{~h}$ prior to use. Anhydrous toluene (Aldrich), anhydrous hexanes (Aldrich), anhydrous diethyl ether (Aldrich), anhydrous THF (Aldrich) and hexamethyldisiloxane (Aldrich) were dried over KH for 24 hours, passed through a column of activated alumina and stored over activated $4 \AA$ molecular sieves prior to use. Benzene- $d_{6}$ (Aldrich) and $\mathrm{CDCl}_{3}$ were dried over activated $4 \AA$ molecular sieves prior to use. THF- $d_{8}$ (CIL) was dried over a Na mirror prior to use. $\mathrm{LiCH}_{2} \mathrm{SiMe}_{3}$ was purchased as a solution (1.0M in pentane, Aldrich), which was filtered through Celite and isolated as a 
white powder upon removal of volatiles under dynamic vacuum. [\{2-Br-4-Me- $\left.\left.\mathrm{C}_{6} \mathrm{H}_{3}\right\}_{2} \mathrm{~N}\right] \mathrm{H}$ and $\left[\left\{2-\left({ }^{i} \mathrm{Pr}_{2} \mathrm{P}\right)-4-\mathrm{Me}-\mathrm{C}_{6} \mathrm{H}_{3}\right\}_{2} \mathrm{~N}\right] \mathrm{H}(\mathbf{1})$ were prepared according to literature procedures. ${ }^{1}$

Synthesis of $\mathrm{Lu}\left(\mathrm{CH}_{2} \mathrm{SiMe}_{3}\right)_{3}(\mathrm{THF})_{2}$. This procedure is slightly modified from the one presented by Okuda and co-workers. ${ }^{2}$ A $125 \mathrm{~mL}$ side-arm flask was charged with $\mathrm{LuCl}_{3}(4.99 \mathrm{~g}, 17.74$ mmol), THF (50 mL) and a magnetic stir bar. The suspension was stirred and heated to $60^{\circ} \mathrm{C}$ for 30 minutes, after which the stirring was stopped and the suspension was allowed to cool to ambient temperature. The mixture was then cooled to $-35^{\circ} \mathrm{C}$ and to the stirring mixture was added $\mathrm{LiCH}_{2} \mathrm{SiMe}_{3}(5.01 \mathrm{~g}, 53.21 \mathrm{mmol})$ and the mixture allowed to warm to ambient temperature and stirred overnight to yield a clear, colorless solution. The volatiles were removed under reduced pressure to give a white colored oil. The oil was stirred with pentane $(75 \mathrm{~mL})$ for one hour and the pentane solution was filtered through a sintered glass filter frit topped with a layer of Celite. The remaining white oil was washed again with pentane $(50 \mathrm{~mL})$ and the solution was filtered again. Removal of pentane under reduced pressure gave $\mathrm{Lu}\left(\mathrm{CH}_{2} \mathrm{SiMe}_{3}\right)_{3}(\mathrm{THF})_{2}$ as a fluffy, white solid (5.856 g, 57\%). ${ }^{1} \mathrm{H}$ NMR data match those reported in the literature. ${ }^{2}{ }^{1} \mathrm{H}$ NMR $\left(\mathrm{C}_{6} \mathrm{D}_{6}, 298 \mathrm{~K}\right): \delta 3.95$ (m, 8H, THF), 1.31 (m, 8H, THF), 0.29 (s, 27H, $\left.\mathrm{CH}_{2} \mathrm{SiMe}_{3}\right),-0.90$ (s, 6H, $\left.\mathrm{CH}_{2} \mathrm{SiMe}_{3}\right) .{ }^{13} \mathrm{C}\left\{{ }^{1} \mathrm{H}\right\}$ NMR $\left(\mathrm{C}_{6} \mathrm{D}_{6}, 298 \mathrm{~K}\right): \delta 71.28\left(\mathrm{~s}, \mathrm{CH}_{2}-\mathrm{THF}\right), 42.11\left(\mathrm{~s}, \mathrm{CH}_{2} \mathrm{SiMe}_{3}\right)$, 25.42 (s, $\left.\mathrm{CH}_{2}-\mathrm{THF}\right), 5.15$ (s, $\mathrm{CH}_{2} \mathrm{SiMe}_{3}$ ).

Synthesis of $\mathrm{MesPH}_{2}$. This is a modification of an earlier published procedure. ${ }^{3}$ To a cooled ($\left.78{ }^{\circ} \mathrm{C}\right)$ suspension of $\mathrm{LiAlH}_{4}(5.27 \mathrm{~g}, 0.139 \mathrm{~mol})$ in $\mathrm{Et}_{2} \mathrm{O}(150 \mathrm{~mL})$ was added dropwise a solution of $\mathrm{MesPCl}_{2}{ }^{4}(38.40 \mathrm{~g}, 0.173 \mathrm{~mol})$ in $\mathrm{Et}_{2} \mathrm{O}(150 \mathrm{~mL})$. After the addition was complete (ca. $1 \mathrm{~h}$ ), the reaction was warmed to room temperature (ca. $1 \mathrm{~h}$ ). ${ }^{31} \mathrm{P}$ NMR spectroscopy of an aliquot removed from the reaction mixture confirmed that the phosphine had been formed quantitatively. Degassed water (ca. $200 \mathrm{~mL}$ ) was added to quench residual aluminum hydrides. 
(CAUTION: extreme care should be taken when adding the first few $m L$ of water since the quenching is highly exothermic and $\mathrm{H}_{2}$ is evolved). The $\mathrm{Et}_{2} \mathrm{O}$ layer was separated and the aqueous layer was extracted with a further $150 \mathrm{~mL}$ of $\mathrm{Et}_{2} \mathrm{O}$. The organic layers were combined, filtered, dried with $\mathrm{MgSO}_{4}$, and the $\mathrm{Et}_{2} \mathrm{O}$ was removed under reduced pressure. The product was purified by vacuum distillation $\left(65-75^{\circ} \mathrm{C}, 1 \mathrm{~mm} \mathrm{Hg}\right)$. Yield: $17.72 \mathrm{~g}(67 \%)$. CAUTION: The product is pyrophoric and very malodorous. ${ }^{31} \mathrm{P} \mathrm{NMR}\left(\mathrm{C}_{6} \mathrm{D}_{6}\right): \delta-155.4\left(\mathrm{t},{ }^{1} J_{\mathrm{PH}}=204 \mathrm{~Hz}\right) ;{ }^{1} \mathrm{H}$ $\operatorname{NMR}\left(\mathrm{C}_{6} \mathrm{D}_{6}\right): \delta 6.70(\mathrm{~s}, 2 \mathrm{H}, m-\mathrm{Mes}), 3.60\left(\mathrm{~d},{ }^{1} J_{\mathrm{PH}}=203 \mathrm{~Hz}, 2 \mathrm{H},-\mathrm{PH}_{2}\right), 2.21\left(\mathrm{~s}, 6 \mathrm{H}, o-\mathrm{CH}_{3}\right)$, $2.09\left(\mathrm{~s}, 3 \mathrm{H}, p-\mathrm{CH}_{3}\right)$.

Synthesis of $\left[\left\{2-\left(\mathbf{P h}_{2} \mathbf{P}\right)-4-\mathrm{Me}-\mathrm{C}_{6} \mathbf{H}_{3}\right\}_{2} \mathbf{N}\right] \mathbf{H}$ (2). ${ }^{5}$ To a solution of $\left[2-\mathrm{Br}-4-\mathrm{Me}-\mathrm{C}_{6} \mathrm{H}_{3}\right]_{2} \mathrm{NH}(710$ $\mathrm{mg}, 2.0 \mathrm{mmol})$ in $\mathrm{Et}_{2} \mathrm{O}(50 \mathrm{~mL})$ at $-35^{\circ} \mathrm{C}$ was slowly added $n$-BuLi $(2.5 \mathrm{~mL}$ of $2.5 \mathrm{M}$ solution in hexanes, $6.25 \mathrm{mmol}$ ). The mixture was allowed to warm up to ambient temperature and stirred for $3 \mathrm{~h}$, and then it was cooled down to $-35^{\circ} \mathrm{C} . \mathrm{Ph}_{2} \mathrm{PCl}(1.13 \mathrm{~mL}, 6.30 \mathrm{mmol})$ was slowly added to the mixture and it was allowed to warm up to ambient temperature while stirring. After 20 hours, excess aqueous $\mathrm{HCl}(2 \mathrm{~mL})$ was added to the reaction mixture and the mixture was allowed to stir $0.5 \mathrm{~h} . \mathrm{Na}_{2} \mathrm{CO}_{3}$ solid was added to mixture until the $\mathrm{pH}$ value of organic phrase equaled 7. All of the volatiles were removed under reduced pressure, and the residue was extracted with toluene $(50 \mathrm{~mL})$ and the precipitate was filtered off. The filtrate was collected, and the volatiles were removed under reduced pressure. The resulting solid was washed with $2 \mathrm{x}$ $5 \mathrm{~mL}$ cold ethanol and then dried under dynamic vacuum to afford a white solid (760 mg, $68 \%$ yield). ${ }^{1} \mathrm{H}$ NMR $\left(\mathrm{C}_{6} \mathrm{D}_{6}, 298 \mathrm{~K}\right): \delta 7.34(\mathrm{~m}, 8 \mathrm{H}, \mathrm{Ar}-\mathrm{H}), 7.26(\mathrm{~m}, 2 \mathrm{H}, \mathrm{Ar}-\mathrm{H}), 7.08(\mathrm{t}, 1 \mathrm{H}, \mathrm{J}=6 \mathrm{~Hz}$, N-H), 7.01 (m, 12H, Ar-H), $6.92(\mathrm{~m}, 2 \mathrm{H}$, Ar-H), 6.84 (d, 2H, J = 8 Hz, Ar-H), 1.92 (s, 6H). ${ }^{13} \mathrm{C}\left\{{ }^{1} \mathrm{H}\right\}$ NMR $\left(\mathrm{CDCl}_{3}, 298 \mathrm{~K}\right): \delta 144.97\left(\mathrm{~d}, 21.0 \mathrm{~Hz}\right.$, quat $\left.\mathrm{C}_{\mathrm{Ar}}\right), 136.38\left(\mathrm{~d}, 9.1 \mathrm{~Hz}\right.$, quat $\left.\mathrm{C}_{\mathrm{Ar}}\right)$, 
$134.56\left(\mathrm{~s}, \mathrm{C}_{\mathrm{Ar}}\right), 133.70\left(\mathrm{~d}, 20.5 \mathrm{~Hz}, \mathrm{C}_{\mathrm{Ar}}\right), 130.79$ (s, quat $\left.\mathrm{C}_{\mathrm{Ar}}\right), 130.72\left(\mathrm{~s}, \mathrm{C}_{\mathrm{Ar}}\right), 128.69\left(\mathrm{~m}, \mathrm{C}_{\mathrm{Ar}}\right)$, $118.76\left(\mathrm{~s}, \mathrm{C}_{\mathrm{Ar}}\right), 21.95\left(\mathrm{~s}, \mathrm{CH}_{3}\right) .{ }^{31} \mathrm{P}\left\{{ }^{1} \mathrm{H}\right\} \mathrm{NMR}\left(\mathrm{C}_{6} \mathrm{D}_{6}, 298 \mathrm{~K}\right): \delta-18.9$.

Synthesis of $\left[\left\{2-\left({ }^{i} \operatorname{Pr}_{2} \mathrm{P}\right)-4-\mathrm{Me}-\mathrm{C}_{6} \mathrm{H}_{3}\right\}_{2} \mathrm{~N}\right] \mathrm{Lu}\left(\mathrm{CH}_{2} \mathrm{SiMe}_{3}\right)_{2}$ (3). A scintillation vial was charged with $\left[\left\{2-\left({ }^{i} \operatorname{Pr}_{2} \mathrm{P}\right)-4-\mathrm{Me}-\mathrm{C}_{6} \mathrm{H}_{3}\right\}_{2} \mathrm{~N}\right] \mathrm{H}(\mathbf{1})(1.353 \mathrm{~g}, 3.150 \mathrm{mmol})$, a magnetic stir bar, and $5 \mathrm{~mL}$ pentane. To the stirring suspension of 2 was added $\mathrm{Lu}\left(\mathrm{CH}_{2} \mathrm{SiMe}_{3}\right)_{3}(\mathrm{THF})_{2}(1.8276 \mathrm{~g}, 3.150$ mmol) as a solution in pentane $(5 \mathrm{~mL})$. Upon addition the solution began to turn yellow and was stirred overnight to give a yellow solution. The pentane was removed under dynamic vacuum to give a yellow, foamy solid. This was then dissolved in $20 \mathrm{~mL}$ of hot $\left(\mathrm{Me}_{3} \mathrm{Si}\right)_{2} \mathrm{O}$ and filtered through a glass fiber plug to give a clear yellow solution. Upon cooling to ambient temperature and sitting overnight large, yellow blocks formed. The solution was decanted and the crystals were washed with $5 \mathrm{~mL}$ of cold $\left(-35^{\circ} \mathrm{C}\right)$ pentane and dried under dynamic vacuum $(1.379 \mathrm{~g}$, $1.773 \mathrm{mmo}, 56 \%) .{ }^{1} \mathrm{H}$ NMR $\left(\mathrm{C}_{6} \mathrm{D}_{6}, 298 \mathrm{~K}\right): \delta 7.08\left(\mathrm{dd}, 4.3\right.$ and $\left.8.5 \mathrm{~Hz}, 2 \mathrm{H}, \mathrm{C}_{\mathrm{Ar}}-H\right), 6.89(\mathrm{dd}, 6.9$ and $\left.8.7 \mathrm{~Hz}, 2 \mathrm{H}, \mathrm{C}_{\mathrm{Ar}}-\mathrm{H}\right), 6.79\left(\mathrm{br} \mathrm{m}, 2 \mathrm{H}, \mathrm{C}_{\mathrm{Ar}}-\mathrm{H}\right), 2.17\left(\mathrm{~m}, 2 \mathrm{H}, \mathrm{CHMe} \mathrm{M}_{2}\right.$, obscured by the peak at $\delta$ 2.14 ppm), 2.14 (s, 6H, $\mathrm{CH}_{3}$ ), 2.01 (sept, $6.9 \mathrm{~Hz}, \mathrm{CHMe}_{2}$ ), 1.17 (m, 18H, CHMe $), 0.84$ (m, 6H, $\left.\mathrm{CHMe}_{2}\right), 0.36$ (s, 18H, CH $\left.\mathrm{SiMe}_{3}\right),-0.34\left(\mathrm{~d}, 2.6 \mathrm{~Hz}, 4 \mathrm{H}, \mathrm{CH}_{2} \mathrm{SiMe}_{3}\right) .{ }^{13} \mathrm{C}\{\mathrm{H}\} \mathrm{NMR}\left(\mathrm{C}_{6} \mathrm{D}_{6}, 298\right.$ $\mathrm{K}$ ): $\delta 161.01$ (pseudo t, $9.8 \mathrm{~Hz}$, quat $\left.\mathrm{C}_{\mathrm{Ar}}\right), 133.7\left(\mathrm{C}_{\mathrm{Ar}}\right), 133.6\left(\mathrm{C}_{\mathrm{Ar}}\right), 127.88$ (pseudo t, $1.8 \mathrm{~Hz}$, quat $\mathrm{C}_{\mathrm{Ar}}$ ), 121.48 (pseudo t, $3.1 \mathrm{~Hz}, \mathrm{C}_{\mathrm{Ar}}$ ), $115.83\left(\mathrm{dd}, 9.7\right.$ and $11.3 \mathrm{~Hz}$, quat $\mathrm{C}_{\mathrm{Ar}}$ ), $56.70(\mathrm{t}, 7.5 \mathrm{~Hz}$, $\mathrm{CH}_{2} \mathrm{SiMe}_{3}$ ), 25.12 (t, $\left.3.3 \mathrm{~Hz},{ }^{i} \mathrm{Pr}\right), 21.12\left(\mathrm{~s}, \mathrm{CH}_{3}\right), 20.58$ (t, $\left.3.6 \mathrm{~Hz},{ }^{i} \mathrm{Pr}\right), 20.14$ (t, $4.2 \mathrm{~Hz},{ }^{i} \mathrm{Pr}$ ), 20.01 (t, $\left.6.5 \mathrm{~Hz},{ }^{i} \mathrm{Pr}\right), 19.47$ (t, $\left.6.1 \mathrm{~Hz},{ }^{i} \mathrm{Pr}\right), 16.82$ (t, $\left.2.8 \mathrm{~Hz},{ }^{i} \mathrm{Pr}\right), 5.34\left(\mathrm{~s}, \mathrm{CH}_{2} \mathrm{SiMe}\right) .{ }^{31} \mathrm{P}\left\{{ }^{1} \mathrm{H}\right\}$ NMR $\left(\mathrm{C}_{6} \mathrm{D}_{6}, 298 \mathrm{~K}\right): \delta 14.5$ (s). Anal. Calcd. for $\mathrm{C}_{34} \mathrm{H}_{62} \mathrm{LuNP}_{2} \mathrm{Si}_{2}(777.95 \mathrm{~g} / \mathrm{mol}): \mathrm{C}, 52.49 ; \mathrm{H}$, 8.03; N, 1.80. Found: C, 52.44; H, 8.35; N, 1.75. $\mathrm{Mp}=130-131^{\circ} \mathrm{C}$.

Synthesis of $\left[\left\{2-\left(\mathbf{P h}_{2} \mathbf{P}\right)-4-\mathrm{Me}_{-} \mathrm{C}_{6} \mathrm{H}_{3}\right\}_{2} \mathbf{N}\right] \mathrm{Lu}\left(\mathrm{CH}_{2} \mathrm{SiMe}_{3}\right)_{2}$ (4). A $125 \mathrm{~mL}$ side-arm flask was charged with $\mathrm{Lu}\left(\mathrm{CH}_{2} \mathrm{SiMe}_{3}\right)_{3}(\mathrm{THF})_{2}(0.293 \mathrm{~g}, 0.518 \mathrm{mmol})$, a magnetic stir bar, and $20 \mathrm{~mL}$ of 
toluene. To the stirring solution, a suspension of $\left[\left\{2-\left(\mathrm{Ph}_{2} \mathrm{P}\right)-4-\mathrm{Me}^{-} \mathrm{C}_{6} \mathrm{H}_{3}\right\}_{2} \mathrm{~N}\right] \mathrm{H}(2)(1.8276 \mathrm{~g}$, $3.150 \mathrm{mmol})$ in toluene $(20 \mathrm{~mL})$ was added portion-wise. The reaction mixture immediately became yellow in color and after two hours the solvent was removed under dynamic vacuum. The yellow solids were triturated with pentane $(10 \mathrm{~mL})$, the pentane decanted and the yellow powder was dried under dynamic vacuum $(0.340 \mathrm{~g}, 0.372 \mathrm{mmol}, 72 \%) .{ }^{1} \mathrm{H}$ NMR $\left(\mathrm{C}_{6} \mathrm{D}_{6}, 298 \mathrm{~K}\right)$ : $\delta 7.58\left(\mathrm{~m}, 8 \mathrm{H}, o-\mathrm{Ph}_{2} \mathrm{P}\right), 6.81-7.09(\mathrm{~m}, 18 \mathrm{H}, \mathrm{Ar}-\mathrm{H}), 0.22\left(\mathrm{~s}, 18 \mathrm{H}, \mathrm{CH}_{2} \mathrm{SiMe}_{3}\right),-0.08(\mathrm{~s}, 4 \mathrm{H}$, $\left.\mathrm{CH}_{2} \mathrm{SiMe}_{3}\right) .{ }^{13} \mathrm{C}\{\mathrm{H}\}$ NMR $\left(\mathrm{C}_{6} \mathrm{D}_{6}, 298 \mathrm{~K}\right): \delta 158.32$ (pseudo t, $11.2 \mathrm{~Hz}$, quat $\left.\mathrm{C}_{\mathrm{Ar}}\right), 134.96(\mathrm{~s}$, $\mathrm{C}_{\mathrm{Ar}}$ ), 134.50 (pseudo t, $7.3 \mathrm{~Hz}, \mathrm{C}_{\mathrm{Ar}}$ ), 134.27 (s, $\mathrm{C}_{\mathrm{Ar}}$ ), 132.97 (pseudo t, $9.4 \mathrm{~Hz}$, quat $\mathrm{C}_{\mathrm{Ar}}$ ), 130.38 (s, $\mathrm{C}_{\mathrm{Ar}}$ ), 129.52 (pseudo t, $2.1 \mathrm{~Hz}$, quat $\mathrm{C}_{\mathrm{Ar}}$ ), 129.34 (pseudo t, $17.9 \mathrm{~Hz}, \mathrm{C}_{\mathrm{Ar}}$ ), $128.65\left(\mathrm{~s}, \mathrm{C}_{\mathrm{Ar}}\right.$ ), 121.26 (pseudo t, $2.8 \mathrm{~Hz}, \mathrm{C}_{\mathrm{Ar}}$ ), 120.79 (pseudo t, $13.1 \mathrm{~Hz}$, quat $\mathrm{C}_{\mathrm{Ar}}$ ), 50.81 (t, $7.8 \mathrm{~Hz}$, $\left.\mathrm{CH}_{2} \mathrm{SiMe}_{3}\right), 20.88\left(\mathrm{~s}, \mathrm{CH}_{3}\right), 5.02\left(\mathrm{~s}, \mathrm{CH}_{2} \mathrm{SiMe}_{3}\right) .{ }^{31} \mathrm{P}\left\{{ }^{1} \mathrm{H}\right\} \mathrm{NMR}\left(\mathrm{C}_{6} \mathrm{D}_{6}, 298 \mathrm{~K}\right): \delta-4.8$. Anal. Calcd. for $\mathrm{C}_{46} \mathrm{H}_{54} \mathrm{LuNP}_{2} \mathrm{Si}_{2}(914.01 \mathrm{~g} / \mathrm{mol}): \mathrm{C}, 60.45 ; \mathrm{H}, 5.95 ; \mathrm{N}, 1.53$. Found: $\mathrm{C}, 60.75 ; \mathrm{H}$, 5.96; $\mathrm{N}, 1.92 . \mathrm{Mp}=>230^{\circ} \mathrm{C}$.

Synthesis of $\left[\left\{2-\left({ }^{i} \mathrm{Pr}_{2} \mathrm{P}\right)-4-\mathrm{Me}-\mathrm{C}_{6} \mathrm{H}_{3}\right\}_{2} \mathrm{NLu}\right]_{2}(\mu-\mathrm{PMes})_{2}$ (5). A 50-mL thick-walled Schlenk tube equipped with Teflon valve was charged with a magnetic stir bar, [\{2-( $\left.\operatorname{Pr}_{2} \mathrm{P}\right)-4-\mathrm{Me}-$ $\left.\left.\mathrm{C}_{6} \mathrm{H}_{3}\right\}_{2} \mathrm{~N}\right] \mathrm{Lu}\left(\mathrm{CH}_{2} \mathrm{SiMe}_{3}\right)_{2}$ (3) $(0.9645 \mathrm{~g}, 1.336 \mathrm{mmol})$, and a solution of $\mathrm{MesPH}_{2}(0.2034 \mathrm{~g}, 1.336$ $\mathrm{mmol})$ in toluene $(5 \mathrm{~mL})$. The yellow solution was then stirred overnight in a silicone oil bath $\left(80^{\circ} \mathrm{C}\right)$ to yield a cherry red colored solution. Removal of volatiles afforded a red solid, which was then triturated with hexanes $(2 \times 5 \mathrm{~mL})$ to yield a fine red precipitate upon drying using dynamic vacuum. The free flowing red powder was dissolved in hot toluene $(5 \mathrm{~mL})$. Upon cooling, the toluene solution was layered with pentane $(5 \mathrm{~mL})$ yielding red crystals of compound 5 (0.5203g, $0.3452 \mathrm{mmol}, 52 \%) .{ }^{1} \mathrm{H}$ NMR $\left(\mathrm{C}_{6} \mathrm{D}_{6}, 298 \mathrm{~K}\right): \delta 6.95-7.25\left(\mathrm{~m}, 12 \mathrm{H}, \mathrm{C}_{\mathrm{Ar}}-\mathrm{H}\right), 6.79(\mathrm{~s}$, $\left.4 \mathrm{H}, \mathrm{C}_{\mathrm{Ar}}-\mathrm{H}\right), 2.26\left(\mathrm{~m}, 8 \mathrm{H}, \mathrm{CHMe}_{2}\right), 2.19\left(\mathrm{~s}, 12 \mathrm{H}, \mathrm{CH}_{3}\right), 2.14\left(\mathrm{~s}, 12 \mathrm{H}, \mathrm{CH}_{3}\right), 2.12\left(\mathrm{~s}, 6 \mathrm{H}, \mathrm{CH}_{3}\right)$, 
1.39 (pseudo pentet, 24H, CHMe $e_{2}$ ), 1.13 (pseudo q, 24H, $\mathrm{CHMe}$ ), 0.94 (pseudo q, 12H, $\mathrm{CHMe}_{2}$ ). ${ }^{13} \mathrm{C}\{\mathrm{H}\}$ NMR (THF- $d_{8}, 298 \mathrm{~K}$ ): $\delta 162.18$ (pseudo t, $11.2 \mathrm{~Hz}$, quat $\mathrm{C}_{\mathrm{Ar}}$ ), 153.67 (br s, quat $\left.\mathrm{C}_{\mathrm{Ar}}\right), 139.64\left(\mathrm{~s}\right.$, quat $\left.\mathrm{C}_{\mathrm{Ar}}\right), 138.42\left(\mathrm{~s}\right.$, quat $\left.\mathrm{C}_{\mathrm{Ar}}\right), 133.63\left(\mathrm{~s}, \mathrm{C}_{\mathrm{Ar}}\right), 133.14\left(\mathrm{~s}, \mathrm{C}_{\mathrm{Ar}}\right), 129.66(\mathrm{~s}$, $\left.\mathrm{C}_{\mathrm{Ar}}\right), 129.6\left(\mathrm{~s}\right.$, quat $\left.\mathrm{C}_{\mathrm{Ar}}\right), 128.9\left(\mathrm{~s}, \mathrm{C}_{\mathrm{Ar}}\right), 127.43\left(\mathrm{~s}\right.$, quat $\left.\mathrm{C}_{\mathrm{Ar}}\right), 127.32$ (s, quat $\left.\mathrm{C}_{\mathrm{Ar}}\right), 126.04\left(\mathrm{~s}, \mathrm{C}_{\mathrm{Ar}}\right)$, $121.56\left(\mathrm{~s}, \mathrm{C}_{\mathrm{Ar}}\right.$ ), 119.03 (pseudo t, $10.4 \mathrm{~Hz}$, quat $\mathrm{C}_{\mathrm{Ar}}$ ), 26.59 (pseudo t, $5.2 \mathrm{~Hz}, \mathrm{CH}_{3}$ ), 26.05 (br s, $\mathrm{CHMe}_{2}$ ), 21.50 (s, $\mathrm{CH}_{3}$ ), 21.18 (pseudo t, $6.0 \mathrm{~Hz}, \mathrm{CHMe}_{2}$ ), 20.82 (s, $\left.\mathrm{CH}_{3}\right), 20.77$ (s, $\mathrm{CH}_{3}$ ), 20.52 (br s, $\mathrm{CH}_{3}$ ), 20.08 (pseudo t, $4.9 \mathrm{~Hz}, \mathrm{CH}_{3}$ ), 19.34 (br s, $\mathrm{CH}_{3}$ ), 17.92 ( br s, $\mathrm{CH}_{3}$ ). ${ }^{31} \mathrm{P}\left\{{ }^{1} \mathrm{H}\right\} \mathrm{NMR}$ $\left(\mathrm{C}_{6} \mathrm{D}_{6}, 298 \mathrm{~K}\right): \delta 186.8$ (pentet, $\left.{ }^{2} \mathrm{~J}_{\mathrm{PP}}=14.6 \mathrm{~Hz}\right), 18.11\left(\mathrm{t},{ }^{2} \mathrm{~J}_{\mathrm{PP}}=14.6 \mathrm{~Hz}\right)$. Anal. Calcd. for $\mathrm{C}_{84} \mathrm{H}_{118} \mathrm{Lu}_{2} \mathrm{~N}_{2} \mathrm{P}_{6}(1691.63 \mathrm{~g} / \mathrm{mol}): \mathrm{C}, 59.64 ; \mathrm{H}, 7.03 ; \mathrm{N}, 1.66$. Found: C, 59.77; H, 7.16; N,1.67. $\mathrm{Mp}=245-246^{\circ} \mathrm{C}$.

Procedure for Generation of $\boldsymbol{E}-\mathbf{M e s} P=\mathbf{C}(\mathbf{H})^{t} \mathbf{B u}$. A J-Young NMR tube was charged with $[\{2-$ $\left.\left.\left({ }^{i} \mathrm{Pr}_{2} \mathrm{P}\right)-4-\mathrm{Me}-\mathrm{C}_{6} \mathrm{H}_{3}\right\}_{2} \mathrm{NLu}\right]_{2}(\mu \text {-PMes})_{2}$ (5) $(29.4 \mathrm{mg}, 0.0195 \mathrm{mmol})$ and $0.6 \mathrm{~mL}$ of $\mathrm{C}_{6} \mathrm{D}_{6}$. Pivalaldehyde $(4.2 \mu \mathrm{L}, 3.36 \mathrm{mg}, 0.0390 \mathrm{mmol})$ was injected using a syringe and the tube was agitated. Yields were determined by ${ }^{1} \mathrm{H}$ NMR spectroscopy, using diphenyl methane as an internal standard. ${ }^{1} \mathrm{H}$ and ${ }^{31} \mathrm{P}$ NMR spectra confirmed the formation of $E$-MesP $\mathrm{P} C(\mathrm{H}){ }^{t} \mathrm{Bu}(48 \%$ yield, ${ }^{31}$ P NMR $\delta 227.1$ ppm, lit. $\left.\left(\mathrm{C}_{6} \mathrm{D}_{6}\right) \delta 228.4 \mathrm{ppm}\right){ }^{6}$

Procedure for Generation of $\mathbf{M e s} \mathbf{P}=\mathbf{C P h} \mathbf{h}_{2}$. A J-Young NMR tube was charged with $[\{2-$ $\left.\left.\left({ }^{i} \mathrm{Pr}_{2} \mathrm{P}\right)-4-\mathrm{Me}-\mathrm{C}_{6} \mathrm{H}_{3}\right\}_{2} \mathrm{NLu}\right]_{2}(\mu-\mathrm{PMes})_{2}$ (5) $(37.3 \mathrm{mg}, 0.02474 \mathrm{mmol})$ and $0.4 \mathrm{~mL}$ of $\mathrm{C}_{6} \mathrm{D}_{6}$. Benzophenone $(9.0 \mathrm{mg}, 0.04948 \mathrm{mmol})$ was added as a solution in $\mathrm{C}_{6} \mathrm{D}_{6}(0.2 \mathrm{~mL})$ and the tube was agitated. Yields were determined by ${ }^{1} \mathrm{H}$ NMR spectroscopy, using diphenyl methane as an internal standard. ${ }^{1} \mathrm{H}$ and ${ }^{31} \mathrm{P}$ NMR spectra confirmed the formation of $\mathrm{Mes} \mathrm{P}=\mathrm{CPh}_{2}(72 \%$ yield, ${ }^{31} \mathrm{P}$ NMR $\delta 234.0$ ppm, lit. $\left.\left(\mathrm{CDCl}_{3}\right) \delta 233.06 \mathrm{ppm}\right){ }^{7}$ 
Generation of Phosphaindole 6. A J-Young NMR tube was charged with [ $\left\{2-\left({ }^{i} \operatorname{Pr}_{2} \mathrm{P}\right)-4-\mathrm{Me}-\right.$ $\left.\left.\mathrm{C}_{6} \mathrm{H}_{3}\right\}_{2} \mathrm{~N}\right] \mathrm{Lu}\left(\mathrm{CH}_{2} \mathrm{SiMe}_{3}\right)_{2}(3)(58.5 \mathrm{mg}, 0.0640 \mathrm{mmol})$ and a solution of $\mathrm{H}_{2} \mathrm{P}-2,4,6-{ }^{t} \mathrm{Bu}_{3}-\mathrm{C}_{6} \mathrm{H}_{2}$ (15.9 mg, $0.0640 \mathrm{mmol})$ in $\mathrm{C}_{6} \mathrm{D}_{6}(0.6 \mathrm{~mL})$ was added. The mixture was heated to $80^{\circ} \mathrm{C}$ for 7 hours to give quantitative formation of 6 as monitored by ${ }^{31} \mathrm{P}$ NMR spectroscopy $\left({ }^{31} \mathrm{P}\right.$ NMR $\delta$ $79.14 \mathrm{ppm}\left(\mathrm{dm},{ }^{1} \mathrm{~J}_{\mathrm{PH}}=181.1 \mathrm{~Hz}\right)$, lit. $\left(\mathrm{C}_{6} \mathrm{D}_{6}\right) \delta 78.4 \mathrm{ppm}\left(\mathrm{dm},{ }^{1} \mathrm{~J}_{\mathrm{PH}}=181.9 \mathrm{~Hz}\right) .{ }^{1} \mathrm{H}$ and ${ }^{31} \mathrm{P} \mathrm{NMR}$ spectroscopic data match the literature. ${ }^{8}$ 


\section{Crystallographic Details for Complexes 3 and 5 (Tables S1-S7).}

Single crystals of complexes $\mathbf{3}$ and $\mathbf{5}$ were mounted from Paratone N oil (Hampton Research) onto a nylon cryoloop under argon gas flow and placed on a Bruker SMART APEX II CCD diffractometer (Compund 3), or a Bruker Platform with 1k CCD, each equipped with a KRYOFLEX liquid nitrogen vapor cooling device. The instruments were equipped with a graphite monochromatized MoK $\alpha$ X-ray source $(\lambda=0.71073 \AA)$. A hemisphere of data was collected using $\omega$ scans, with 5 second frame exposure (3) or 10 second frame exposure times (5) and $0.3^{\circ}$ frame widths. Data collection and initial indexing and cell refinement were handled using APEX II software. ${ }^{9}$ Frame integration, including Lorentz-polarization corrections and final cell parameter calculations were carried out using SAINT+ software. ${ }^{10}$ The data were corrected for absorption using the SADABS program. ${ }^{11}$ Decay of reflection intensity was monitored by analysis of redundant frames. The structures of $\mathbf{3}$ and $\mathbf{5}$ were solved using direct methods. Structure solution and refinement were performed using SHELXTL. ${ }^{12}$ The figures for both complexes were made using ORTEP-3 for Windows. ${ }^{13}$

All non-hydrogen atoms were refined anisotropically, and hydrogen atoms were treated as idealized contributions. Two ${ }^{i} \operatorname{Pr}$ groups (C19-C21, C22-C24) of 5 were disordered and refined in two one-half occupancy positions. Hydrogen atoms were not included on these atoms for refinement. The SQUEEZE feature in Platon $^{14}$ was implemented to take into account the disordered solvent in compound 5 (2 equivalents of toluene). 
Table S1. Crystal Data and Structure Refinement for $\left.\left[2-\left({ }^{i} \mathrm{Pr}_{2} \mathrm{P}\right)-4-\mathrm{Me}_{6} \mathrm{C}_{6} \mathrm{H}_{3}\right\}_{2} \mathrm{~N}\right] \mathrm{Lu}\left(\mathrm{CH}_{2} \mathrm{SiMe}_{3}\right)_{2}(3)$.

Identification code

Empirical formula

Formula weight

Temperature

Wavelength

Crystal system

Space group

Unit cell dimensions

Volume

Z

Density (calculated)

Absorption coefficient

$\mathrm{F}(000)$

Crystal size

$\Theta$ Range for data collection

Index ranges

Reflections collected

Independent reflections

Completeness to $\Theta=24.59^{\circ}$

Max. and min. transmission

Refinement method

Data / restraints / parameters

Goodness-of-fit on $\mathrm{F}^{2}$

Final $\mathrm{R}$ indices $[\mathrm{I}>2 \sigma(\mathrm{I})]$

$\mathrm{R}$ indices (all data)

Largest diff. peak and hole apx 525

$\mathrm{C}_{34} \mathrm{H}_{62} \mathrm{Lu} \mathrm{N} \mathrm{P} \mathrm{P}_{2} \mathrm{Si}_{2}$

777.94

100(2) K

$0.71073 \AA$

triclinic

P $\overline{1}$

$\mathrm{a}=11.8412(16) \AA$

$\alpha=93.120(2)^{\circ}$

$\mathrm{b}=12.0344(17) \AA$

$\beta=109.3100(1)^{\circ}$

$\mathrm{c}=15.568(2) \AA$

$\gamma=112.1280(1)^{\circ}$

$1898.3(5) \AA^{3}$

2

$1.361 \mathrm{Mg} / \mathrm{m}^{3}$

$2.770 \mathrm{~mm}^{-1}$

804

$0.18 \times 0.12 \times 0.04 \mathrm{~mm}^{3}$

2.01 to $24.59^{\circ}$.

$-13 \leq \mathrm{h} \leq 13,-14 \leq \mathrm{k} \leq 14,-18 \leq 1 \leq 18$

17078

$6353[\mathrm{R}(\mathrm{int})=0.0583]$

$99.4 \%$

0.8973 and 0.6355

Full-matrix least-squares on $\mathrm{F}^{2}$

6353 / 0 / 377

0.967

$\mathrm{R} 1=0.0409, \mathrm{wR} 2=0.0827$

$\mathrm{R} 1=0.0575, \mathrm{wR} 2=0.0882$

1.689 and -1.503 e. $\AA^{-3}$ 
Table S2. Bond Lengths $[\AA]$ for $\left.\left[2-\left({ }^{i} \mathrm{Pr}_{2} \mathrm{P}\right)-4-\mathrm{Me}_{-} \mathrm{C}_{6} \mathrm{H}_{3}\right\}_{2} \mathrm{~N}\right] \mathrm{Lu}\left(\mathrm{CH}_{2} \mathrm{SiMe}_{3}\right)_{2}(3)$.

\begin{tabular}{|c|c|c|c|}
\hline Lu(1)-N(1) & $2.244(5)$ & $\mathrm{C}(9)-\mathrm{C}(11)$ & $1.515(8)$ \\
\hline $\mathrm{Lu}(1)-\mathrm{C}(5)$ & $2.307(6)$ & $\mathrm{C}(12)-\mathrm{C}(13)$ & $1.509(8)$ \\
\hline $\mathrm{Lu}(1)-\mathrm{C}(1)$ & $2.322(6)$ & $C(12)-C(14)$ & $1.514(8)$ \\
\hline $\mathrm{Lu}(1)-\mathrm{P}(1)$ & $2.8534(15)$ & $C(15)-C(16)$ & $1.516(8)$ \\
\hline $\mathrm{Lu}(1)-\mathrm{P}(2)$ & $2.8547(15)$ & $C(15)-C(17)$ & $1.528(8)$ \\
\hline $\mathrm{N}(1)-\mathrm{C}(33)$ & $1.395(7)$ & $C(18)-C(19)$ & $1.510(8)$ \\
\hline $\mathrm{N}(1)-\mathrm{C}(26)$ & $1.400(7)$ & $\mathrm{C}(18)-\mathrm{C}(20)$ & $1.522(7)$ \\
\hline $\mathrm{P}(1)-\mathrm{C}(21)$ & $1.823(6)$ & $C(21)-C(22)$ & $1.394(7)$ \\
\hline $\mathrm{P}(1)-\mathrm{C}(9)$ & $1.839(6)$ & $\mathrm{C}(21)-\mathrm{C}(26)$ & $1.402(8)$ \\
\hline $\mathrm{P}(1)-\mathrm{C}(12)$ & $1.846(6)$ & $\mathrm{C}(22)-\mathrm{C}(23)$ & $1.378(7)$ \\
\hline $\mathrm{P}(2)-\mathrm{C}(28)$ & $1.809(6)$ & $\mathrm{C}(23)-\mathrm{C}(24)$ & $1.383(8)$ \\
\hline $\mathrm{P}(2)-\mathrm{C}(15)$ & $1.842(6)$ & $\mathrm{C}(23)-\mathrm{C}(27)$ & $1.493(7)$ \\
\hline $\mathrm{P}(2)-\mathrm{C}(18)$ & $1.856(6)$ & $\mathrm{C}(24)-\mathrm{C}(25)$ & $1.372(7)$ \\
\hline $\operatorname{Si}(1)-C(1)$ & $1.817(6)$ & $C(25)-C(26)$ & $1.397(7)$ \\
\hline $\mathrm{Si}(1)-\mathrm{C}(3)$ & $1.863(7)$ & $\mathrm{C}(28)-\mathrm{C}(29)$ & $1.386(8)$ \\
\hline $\operatorname{Si}(1)-C(4)$ & $1.865(6)$ & $\mathrm{C}(28)-\mathrm{C}(33)$ & $1.407(7)$ \\
\hline $\mathrm{Si}(1)-\mathrm{C}(2)$ & $1.873(7)$ & $\mathrm{C}(29)-\mathrm{C}(30)$ & $1.392(8)$ \\
\hline $\operatorname{Si}(2)-C(5)$ & $1.835(6)$ & $\mathrm{C}(30)-\mathrm{C}(31)$ & $1.378(7)$ \\
\hline $\operatorname{Si}(2)-C(6)$ & $1.862(6)$ & $\mathrm{C}(30)-\mathrm{C}(34)$ & $1.494(7)$ \\
\hline $\operatorname{Si}(2)-C(7)$ & $1.864(7)$ & $\mathrm{C}(31)-\mathrm{C}(32)$ & $1.370(7)$ \\
\hline $\mathrm{Si}(2)-\mathrm{C}(8)$ & $1.867(6)$ & $\mathrm{C}(32)-\mathrm{C}(33)$ & $1.409(7)$ \\
\hline C(9)-C(10) & $1.508(8)$ & & \\
\hline
\end{tabular}


Table S3. Bond Angles [ $\left.{ }^{\circ}\right]$ for $\left.\left[2-\left({ }^{i} \mathrm{Pr}_{2} \mathrm{P}\right)-4-\mathrm{Me}-\mathrm{C}_{6} \mathrm{H}_{3}\right\}_{2} \mathrm{~N}\right] \mathrm{Lu}\left(\mathrm{CH}_{2} \mathrm{SiMe}_{3}\right)_{2}(3)$.

\begin{tabular}{|c|c|c|c|}
\hline $\mathrm{N}(1)-\mathrm{Lu}(1)-\mathrm{C}(5)$ & $112.82(18)$ & $\mathrm{C}(5)-\mathrm{Si}(2)-\mathrm{C}(8)$ & $110.4(3)$ \\
\hline $\mathrm{N}(1)-\mathrm{Lu}(1)-\mathrm{C}(1)$ & $128.69(19)$ & $\mathrm{C}(6)-\mathrm{Si}(2)-\mathrm{C}(8)$ & $107.0(3)$ \\
\hline$C(5)-\operatorname{Lu}(1)-C(1)$ & $118.0(2)$ & $C(7)-\operatorname{Si}(2)-C(8)$ & $107.3(3)$ \\
\hline $\mathrm{N}(1)-\mathrm{Lu}(1)-\mathrm{P}(1)$ & $70.37(12)$ & $\operatorname{Si}(1)-C(1)-\operatorname{Lu}(1)$ & $126.2(3)$ \\
\hline $\mathrm{C}(5)-\mathrm{Lu}(1)-\mathrm{P}(1)$ & $103.06(15)$ & $\operatorname{Si}(2)-C(5)-\operatorname{Lu}(1)$ & $127.6(3)$ \\
\hline $\mathrm{C}(1)-\mathrm{Lu}(1)-\mathrm{P}(1)$ & $103.93(14)$ & $C(10)-C(9)-C(11)$ & $111.5(5)$ \\
\hline $\mathrm{N}(1)-\mathrm{Lu}(1)-\mathrm{P}(2)$ & $69.31(11)$ & $\mathrm{C}(10)-\mathrm{C}(9)-\mathrm{P}(1)$ & $109.7(4)$ \\
\hline$C(5)-\operatorname{Lu}(1)-P(2)$ & $101.75(15)$ & $\mathrm{C}(11)-\mathrm{C}(9)-\mathrm{P}(1)$ & $112.1(4)$ \\
\hline $\mathrm{C}(1)-\mathrm{Lu}(1)-\mathrm{P}(2)$ & $93.13(14)$ & $C(13)-C(12)-C(14)$ & $112.1(5)$ \\
\hline $\mathrm{P}(1)-\mathrm{Lu}(1)-\mathrm{P}(2)$ & $138.32(4)$ & $\mathrm{C}(13)-\mathrm{C}(12)-\mathrm{P}(1)$ & $111.0(4)$ \\
\hline $\mathrm{C}(33)-\mathrm{N}(1)-\mathrm{C}(26)$ & $117.3(4)$ & $\mathrm{C}(14)-\mathrm{C}(12)-\mathrm{P}(1)$ & $113.4(4)$ \\
\hline $\mathrm{C}(33)-\mathrm{N}(1)-\mathrm{Lu}(1)$ & $126.4(3)$ & $C(16)-C(15)-C(17)$ & $110.6(5)$ \\
\hline $\mathrm{C}(26)-\mathrm{N}(1)-\mathrm{Lu}(1)$ & $116.2(3)$ & $\mathrm{C}(16)-\mathrm{C}(15)-\mathrm{P}(2)$ & $111.6(4)$ \\
\hline $\mathrm{C}(21)-\mathrm{P}(1)-\mathrm{C}(9)$ & $104.9(3)$ & $\mathrm{C}(17)-\mathrm{C}(15)-\mathrm{P}(2)$ & $108.6(4)$ \\
\hline $\mathrm{C}(21)-\mathrm{P}(1)-\mathrm{C}(12)$ & 105.1(3) & $\mathrm{C}(19)-\mathrm{C}(18)-\mathrm{C}(20)$ & $110.3(5)$ \\
\hline $\mathrm{C}(9)-\mathrm{P}(1)-\mathrm{C}(12)$ & $104.2(3)$ & $\mathrm{C}(19)-\mathrm{C}(18)-\mathrm{P}(2)$ & $114.7(4)$ \\
\hline $\mathrm{C}(21)-\mathrm{P}(1)-\mathrm{Lu}(1)$ & $90.10(17)$ & $\mathrm{C}(20)-\mathrm{C}(18)-\mathrm{P}(2)$ & $110.3(4)$ \\
\hline $\mathrm{C}(9)-\mathrm{P}(1)-\mathrm{Lu}(1)$ & $127.3(2)$ & $C(22)-C(21)-C(26)$ & $119.9(5)$ \\
\hline$C(12)-P(1)-L u(1)$ & $120.4(2)$ & $\mathrm{C}(22)-\mathrm{C}(21)-\mathrm{P}(1)$ & $122.5(4)$ \\
\hline $\mathrm{C}(28)-\mathrm{P}(2)-\mathrm{C}(15)$ & $106.4(3)$ & $\mathrm{C}(26)-\mathrm{C}(21)-\mathrm{P}(1)$ & $117.5(4)$ \\
\hline $\mathrm{C}(28)-\mathrm{P}(2)-\mathrm{C}(18)$ & $103.9(3)$ & $\mathrm{C}(23)-\mathrm{C}(22)-\mathrm{C}(21)$ & $122.5(5)$ \\
\hline $\mathrm{C}(15)-\mathrm{P}(2)-\mathrm{C}(18)$ & $104.4(3)$ & $C(22)-C(23)-C(24)$ & $117.1(5)$ \\
\hline $\mathrm{C}(28)-\mathrm{P}(2)-\mathrm{Lu}(1)$ & $96.59(18)$ & $\mathrm{C}(22)-\mathrm{C}(23)-\mathrm{C}(27)$ & $121.4(5)$ \\
\hline $\mathrm{C}(15)-\mathrm{P}(2)-\mathrm{Lu}(1)$ & $125.9(2)$ & $\mathrm{C}(24)-\mathrm{C}(23)-\mathrm{C}(27)$ & $121.5(5)$ \\
\hline $\mathrm{C}(18)-\mathrm{P}(2)-\mathrm{Lu}(1)$ & $116.63(18)$ & $\mathrm{C}(25)-\mathrm{C}(24)-\mathrm{C}(23)$ & $121.8(5)$ \\
\hline $\mathrm{C}(1)-\mathrm{Si}(1)-\mathrm{C}(3)$ & $111.9(3)$ & $\mathrm{C}(24)-\mathrm{C}(25)-\mathrm{C}(26)$ & $121.6(5)$ \\
\hline $\mathrm{C}(1)-\mathrm{Si}(1)-\mathrm{C}(4)$ & $115.2(3)$ & $\mathrm{C}(25)-\mathrm{C}(26)-\mathrm{N}(1)$ & $122.5(5)$ \\
\hline $\mathrm{C}(3)-\mathrm{Si}(1)-\mathrm{C}(4)$ & 105.2(3) & $C(25)-C(26)-C(21)$ & $117.1(5)$ \\
\hline $\mathrm{C}(1)-\mathrm{Si}(1)-\mathrm{C}(2)$ & $108.4(3)$ & $\mathrm{N}(1)-\mathrm{C}(26)-\mathrm{C}(21)$ & $120.3(5)$ \\
\hline $\mathrm{C}(3)-\mathrm{Si}(1)-\mathrm{C}(2)$ & $107.9(3)$ & $\mathrm{C}(29)-\mathrm{C}(28)-\mathrm{C}(33)$ & $120.4(5)$ \\
\hline $\mathrm{C}(4)-\mathrm{Si}(1)-\mathrm{C}(2)$ & $108.0(3)$ & $\mathrm{C}(29)-\mathrm{C}(28)-\mathrm{P}(2)$ & $123.5(4)$ \\
\hline $\mathrm{C}(5)-\mathrm{Si}(2)-\mathrm{C}(6)$ & $112.5(3)$ & $\mathrm{C}(33)-\mathrm{C}(28)-\mathrm{P}(2)$ & $116.1(4)$ \\
\hline $\mathrm{C}(5)-\mathrm{Si}(2)-\mathrm{C}(7)$ & $113.3(3)$ & $\mathrm{C}(28)-\mathrm{C}(29)-\mathrm{C}(30)$ & $122.2(5)$ \\
\hline$C(6)-S i(2)-C(7)$ & $106.0(3)$ & $\mathrm{C}(31)-\mathrm{C}(30)-\mathrm{C}(29)$ & $116.9(5)$ \\
\hline
\end{tabular}




$\begin{array}{ll}\mathrm{C}(31)-\mathrm{C}(30)-\mathrm{C}(34) & 120.7(5) \\ \mathrm{C}(29)-\mathrm{C}(30)-\mathrm{C}(34) & 122.3(5) \\ \mathrm{C}(32)-\mathrm{C}(31)-\mathrm{C}(30) & 122.2(5) \\ \mathrm{C}(31)-\mathrm{C}(32)-\mathrm{C}(33) & 121.4(5) \\ \mathrm{N}(1)-\mathrm{C}(33)-\mathrm{C}(28) & 120.3(5) \\ \mathrm{N}(1)-\mathrm{C}(33)-\mathrm{C}(32) & 123.0(5) \\ \mathrm{C}(28)-\mathrm{C}(33)-\mathrm{C}(32) & 116.6(5)\end{array}$


Table S4. Crystal Data and Structure Refinement for $\left[\left\{2-\left({ }^{i} \mathrm{Pr}_{2} \mathrm{P}\right)-4-\mathrm{Me}-\mathrm{C}_{6} \mathrm{H}_{3}\right\}_{2} \mathrm{NLu}\right]_{2}(\mu-\mathrm{PMes})_{2}(5)$.

\begin{tabular}{|c|c|c|}
\hline Identification code & \multicolumn{2}{|l|}{ xpl041s } \\
\hline Empirical formula & \multicolumn{2}{|l|}{$\mathrm{C}_{84} \mathrm{H}_{118} \mathrm{Lu}_{2} \mathrm{~N}_{2} \mathrm{P}_{6}$} \\
\hline Formula weight & \multicolumn{2}{|l|}{1691.56} \\
\hline Temperature & \multicolumn{2}{|l|}{$141(2) \mathrm{K}$} \\
\hline Wavelength & \multicolumn{2}{|l|}{$0.71073 \AA$} \\
\hline Crystal system & \multicolumn{2}{|l|}{ monoclinic } \\
\hline Space group & \multicolumn{2}{|l|}{$\mathrm{P} 2{ }_{1} / \mathrm{c}$} \\
\hline \multirow[t]{3}{*}{ Unit cell dimensions } & $\mathrm{a}=19.993(3) \AA$ & $\alpha=90^{\circ}$ \\
\hline & $\mathrm{b}=24.746(4) \AA$ & $\beta=110.892(2)^{\circ}$ \\
\hline & $\mathrm{c}=17.489(3) \AA$ & $\gamma=90^{\circ}$ \\
\hline Volume & \multicolumn{2}{|l|}{$8084(2) \AA^{3}$} \\
\hline $\mathrm{Z}$ & \multicolumn{2}{|l|}{4} \\
\hline Density (calculated) & \multicolumn{2}{|l|}{$1.390 \mathrm{Mg} / \mathrm{m}^{3}$} \\
\hline Absorption coefficient & \multicolumn{2}{|l|}{$2.590 \mathrm{~mm}^{-1}$} \\
\hline $\mathrm{F}(000)$ & \multicolumn{2}{|l|}{3472} \\
\hline Crystal size & \multicolumn{2}{|c|}{$0.10 \times 0.08 \times 0.05 \mathrm{~mm}^{3}$} \\
\hline$\Theta$ Range for data collection & \multicolumn{2}{|l|}{1.49 to $25.02^{\circ}$} \\
\hline Index ranges & \multicolumn{2}{|c|}{$-23 \leq \mathrm{h} \leq 23,-29 \leq \mathrm{k} \leq 29,-20 \leq 1 \leq 20$} \\
\hline Reflections collected & \multicolumn{2}{|l|}{77845} \\
\hline Independent reflections & \multicolumn{2}{|c|}{$14243[\mathrm{R}(\mathrm{int})=0.0794]$} \\
\hline Completeness to $\Theta=25.00^{\circ}$ & \multicolumn{2}{|l|}{$99.9 \%$} \\
\hline Max. and min. transmission & \multicolumn{2}{|c|}{0.8880 and 0.7799} \\
\hline Refinement method & \multicolumn{2}{|c|}{ Full-matrix least-squares on $\mathrm{F}^{2}$} \\
\hline Data / restraints / parameters & \multicolumn{2}{|l|}{$14243 / 82 / 797$} \\
\hline Goodness-of-fit on $\mathrm{F}^{2}$ & \multicolumn{2}{|l|}{0.946} \\
\hline Final $R$ indices $[I>2 \sigma(I)]$ & \multicolumn{2}{|c|}{$\mathrm{R} 1=0.0367, \mathrm{wR} 2=0.0758$} \\
\hline $\mathrm{R}$ indices (all data) & \multicolumn{2}{|c|}{$\mathrm{R} 1=0.0629, \mathrm{wR} 2=0.0826$} \\
\hline Largest diff. peak and hole & \multicolumn{2}{|c|}{0.759 and -0.787 e. $\AA^{-3}$} \\
\hline
\end{tabular}


Table S5. Bond Lengths $[\AA]$ for $\left[\left\{2-\left({ }^{i} \mathrm{Pr}_{2} \mathrm{P}\right)-4-\mathrm{Me}-\mathrm{C}_{6} \mathrm{H}_{3}\right\}_{2} \mathrm{NLu}\right]_{2}(\mu-\mathrm{PMes})_{2}(\mathbf{5})$.

\begin{tabular}{|c|c|c|c|}
\hline $\mathrm{Lu}(1)-\mathrm{N}(1)$ & $2.296(4)$ & $C(2)-C(7)$ & $1.500(7)$ \\
\hline $\mathrm{Lu}(1)-\mathrm{P}(1)$ & $2.6029(15)$ & $\mathrm{C}(3)-\mathrm{C}(4)$ & $1.383(7)$ \\
\hline $\mathrm{Lu}(1)-\mathrm{P}(2)$ & $2.6723(14)$ & $C(4)-C(5)$ & $1.371(8)$ \\
\hline $\mathrm{Lu}(1)-\mathrm{P}(3)$ & $2.8467(18)$ & $\mathrm{C}(4)-\mathrm{C}(8)$ & $1.506(7)$ \\
\hline $\mathrm{Lu}(1)-\mathrm{P}(4)$ & $2.8562(15)$ & $C(5)-C(6)$ & $1.382(8)$ \\
\hline $\operatorname{Lu}(1)-\operatorname{Lu}(2)$ & $3.9352(5)$ & $\mathrm{C}(6)-\mathrm{C}(9)$ & $1.519(8)$ \\
\hline $\mathrm{Lu}(2)-\mathrm{N}(2)$ & $2.296(4)$ & $\mathrm{C}(10)-\mathrm{C}(11)$ & $1.416(7)$ \\
\hline $\mathrm{Lu}(2)-\mathrm{P}(2)$ & $2.5976(14)$ & $C(10)-C(15)$ & $1.424(7)$ \\
\hline $\mathrm{Lu}(2)-\mathrm{P}(1)$ & $2.6526(15)$ & $\mathrm{C}(11)-\mathrm{C}(12)$ & $1.382(7)$ \\
\hline $\mathrm{Lu}(2)-\mathrm{P}(6)$ & $2.8303(14)$ & $C(11)-C(16)$ & $1.507(7)$ \\
\hline $\mathrm{Lu}(2)-\mathrm{P}(5)$ & $2.8780(14)$ & $C(12)-C(13)$ & $1.389(7)$ \\
\hline $\mathrm{P}(1)-\mathrm{C}(1)$ & $1.809(5)$ & $C(13)-C(14)$ & $1.385(7)$ \\
\hline $\mathrm{P}(2)-\mathrm{C}(10)$ & $1.823(5)$ & $\mathrm{C}(13)-\mathrm{C}(17)$ & $1.513(7)$ \\
\hline $\mathrm{P}(3)-\mathrm{C}\left(22^{\prime}\right)$ & $1.769(15)$ & $\mathrm{C}(14)-\mathrm{C}(15)$ & $1.381(7)$ \\
\hline $\mathrm{P}(3)-\mathrm{C}(36)$ & $1.821(5)$ & $C(15)-C(18)$ & $1.511(7)$ \\
\hline $\mathrm{P}(3)-\mathrm{C}\left(19^{\prime}\right)$ & $1.82(2)$ & $\mathrm{C}(19)-\mathrm{C}(20)$ & $1.513(17)$ \\
\hline $\mathrm{P}(3)-\mathrm{C}(19)$ & $1.945(15)$ & $C(19)-C(21)$ & $1.514(15)$ \\
\hline $\mathrm{P}(3)-\mathrm{C}(22)$ & $2.002(14)$ & $\mathrm{C}\left(19^{\prime}\right)-\mathrm{C}\left(20^{\prime}\right)$ & $1.498(18)$ \\
\hline $\mathrm{P}(4)-\mathrm{C}(43)$ & $1.821(5)$ & $\mathrm{C}\left(19^{\prime}\right)-\mathrm{C}\left(21^{\prime}\right)$ & $1.487(17)$ \\
\hline $\mathrm{P}(4)-\mathrm{C}(25)$ & $1.846(5)$ & $\mathrm{C}(22)-\mathrm{C}(24)$ & $1.531(16)$ \\
\hline $\mathrm{P}(4)-\mathrm{C}(28)$ & $1.871(6)$ & $C(22)-C(23)$ & $1.557(15)$ \\
\hline $\mathrm{P}(5)-\mathrm{C}(62)$ & $1.822(5)$ & $\mathrm{C}\left(22^{\prime}\right)-\mathrm{C}\left(23^{\prime}\right)$ & $1.474(16)$ \\
\hline $\mathrm{P}(5)-\mathrm{C}(54)$ & $1.839(6)$ & $\mathrm{C}\left(22^{\prime}\right)-\mathrm{C}\left(24^{\prime}\right)$ & $1.519(14)$ \\
\hline $\mathrm{P}(5)-\mathrm{C}(51)$ & $1.864(5)$ & $C(25)-C(27)$ & $1.524(7)$ \\
\hline $\mathrm{P}(6)-\mathrm{C}(69)$ & $1.810(5)$ & $C(25)-C(26)$ & $1.531(8)$ \\
\hline $\mathrm{P}(6)-\mathrm{C}(45)$ & $1.862(5)$ & $C(28)-C(29)$ & $1.530(7)$ \\
\hline $\mathrm{P}(6)-\mathrm{C}(48)$ & $1.869(5)$ & $C(28)-C(30)$ & $1.533(7)$ \\
\hline $\mathrm{N}(1)-\mathrm{C}(31)$ & $1.406(6)$ & $C(31)-C(36)$ & $1.392(7)$ \\
\hline $\mathrm{N}(1)-\mathrm{C}(38)$ & $1.425(6)$ & $\mathrm{C}(31)-\mathrm{C}(32)$ & $1.398(7)$ \\
\hline $\mathrm{N}(2)-\mathrm{C}(64)$ & $1.408(6)$ & $\mathrm{C}(32)-\mathrm{C}(33)$ & $1.380(7)$ \\
\hline $\mathrm{N}(2)-\mathrm{C}(57)$ & $1.418(6)$ & $C(33)-C(34)$ & $1.386(8)$ \\
\hline $\mathrm{C}(1)-\mathrm{C}(2)$ & $1.416(7)$ & $C(34)-C(35)$ & $1.383(7)$ \\
\hline$C(1)-C(6)$ & $1.423(7)$ & $C(34)-C(37)$ & $1.531(7)$ \\
\hline $\mathrm{C}(2)-\mathrm{C}(3)$ & $1.391(7)$ & $C(35)-C(36)$ & $1.400(7)$ \\
\hline
\end{tabular}




$\begin{array}{llll}\mathrm{C}(38)-\mathrm{C}(43) & 1.405(7) & \mathrm{C}(57)-\mathrm{C}(58) & 1.407(7) \\ \mathrm{C}(38)-\mathrm{C}(39) & 1.414(7) & \mathrm{C}(57)-\mathrm{C}(62) & 1.413(7) \\ \mathrm{C}(39)-\mathrm{C}(40) & 1.370(7) & \mathrm{C}(58)-\mathrm{C}(59) & 1.373(7) \\ \mathrm{C}(40)-\mathrm{C}(41) & 1.391(7) & \mathrm{C}(59)-\mathrm{C}(60) & 1.396(7) \\ \mathrm{C}(41)-\mathrm{C}(42) & 1.387(7) & \mathrm{C}(60)-\mathrm{C}(61) & 1.367(8) \\ \mathrm{C}(41)-\mathrm{C}(44) & 1.503(7) & \mathrm{C}(60)-\mathrm{C}(63) & 1.498(7) \\ \mathrm{C}(42)-\mathrm{C}(43) & 1.388(7) & \mathrm{C}(61)-\mathrm{C}(62) & 1.405(7) \\ \mathrm{C}(45)-\mathrm{C}(46) & 1.512(7) & \mathrm{C}(64)-\mathrm{C}(65) & 1.415(6) \\ \mathrm{C}(45)-\mathrm{C}(47) & 1.519(7) & \mathrm{C}(64)-\mathrm{C}(69) & 1.420(6) \\ \mathrm{C}(48)-\mathrm{C}(49) & 1.523(7) & \mathrm{C}(65)-\mathrm{C}(66) & 1.383(7) \\ \mathrm{C}(48)-\mathrm{C}(50) & 1.525(7) & \mathrm{C}(66)-\mathrm{C}(67) & 1.402(7) \\ \mathrm{C}(51)-\mathrm{C}(52) & 1.533(7) & \mathrm{C}(67)-\mathrm{C}(68) & 1.379(7) \\ \mathrm{C}(51)-\mathrm{C}(53) & 1.534(7) & \mathrm{C}(67)-\mathrm{C}(70) & 1.510(7) \\ \mathrm{C}(54)-\mathrm{C}(56) & 1.523(8) & \mathrm{C}(68)-\mathrm{C}(69) & 1.409(6) \\ \mathrm{C}(54)-\mathrm{C}(55) & 1.533(8) & & \end{array}$


Table S6. Bond Angles $\left[^{\circ}\right]$ for $\left[\left\{2-\left({ }^{i} \mathrm{Pr}_{2} \mathrm{P}\right)-4-\mathrm{Me}-\mathrm{C}_{6} \mathrm{H}_{3}\right\}_{2} \mathrm{NLu}\right]_{2}(\mu-\mathrm{PMes})_{2}(5)$.

\begin{tabular}{|c|c|c|c|}
\hline $\mathrm{N}(1)-\mathrm{Lu}(1)-\mathrm{P}(1)$ & $132.57(10)$ & $\mathrm{C}(10)-\mathrm{P}(2)-\mathrm{Lu}(1)$ & $118.46(17)$ \\
\hline $\mathrm{N}(1)-\mathrm{Lu}(1)-\mathrm{P}(2)$ & $144.50(10)$ & $\mathrm{Lu}(2)-\mathrm{P}(2)-\mathrm{Lu}(1)$ & $96.61(4)$ \\
\hline $\mathrm{P}(1)-\mathrm{Lu}(1)-\mathrm{P}(2)$ & $82.91(4)$ & $\mathrm{C}\left(22^{\prime}\right)-\mathrm{P}(3)-\mathrm{C}(36)$ & $111.4(5)$ \\
\hline $\mathrm{N}(1)-\mathrm{Lu}(1)-\mathrm{P}(3)$ & $67.99(11)$ & $\mathrm{C}\left(22^{\prime}\right)-\mathrm{P}(3)-\mathrm{C}\left(19^{\prime}\right)$ & $75.3(9)$ \\
\hline $\mathrm{P}(1)-\mathrm{Lu}(1)-\mathrm{P}(3)$ & $100.76(6)$ & $\mathrm{C}(36)-\mathrm{P}(3)-\mathrm{C}\left(19^{\prime}\right)$ & $107.5(7)$ \\
\hline $\mathrm{P}(2)-\mathrm{Lu}(1)-\mathrm{P}(3)$ & $112.26(5)$ & $\mathrm{C}\left(22^{\prime}\right)-\mathrm{P}(3)-\mathrm{C}(19)$ & $97.9(8)$ \\
\hline $\mathrm{N}(1)-\mathrm{Lu}(1)-\mathrm{P}(4)$ & $69.00(10)$ & $\mathrm{C}(36)-\mathrm{P}(3)-\mathrm{C}(19)$ & $106.6(6)$ \\
\hline $\mathrm{P}(1)-\mathrm{Lu}(1)-\mathrm{P}(4)$ & $110.07(5)$ & $\mathrm{C}\left(19^{\prime}\right)-\mathrm{P}(3)-\mathrm{C}(19)$ & $23.9(7)$ \\
\hline $\mathrm{P}(2)-\mathrm{Lu}(1)-\mathrm{P}(4)$ & $101.02(4)$ & $\mathrm{C}\left(22^{\prime}\right)-\mathrm{P}(3)-\mathrm{C}(22)$ & $29.1(6)$ \\
\hline $\mathrm{P}(3)-\mathrm{Lu}(1)-\mathrm{P}(4)$ & $136.91(5)$ & $\mathrm{C}(36)-\mathrm{P}(3)-\mathrm{C}(22)$ & $95.7(5)$ \\
\hline $\mathrm{N}(1)-\mathrm{Lu}(1)-\mathrm{Lu}(2)$ & $174.49(10)$ & $\mathrm{C}\left(19^{\prime}\right)-\mathrm{P}(3)-\mathrm{C}(22)$ & $103.4(8)$ \\
\hline $\mathrm{P}(1)-\mathrm{Lu}(1)-\mathrm{Lu}(2)$ & $42.00(3)$ & $\mathrm{C}(19)-\mathrm{P}(3)-\mathrm{C}(22)$ & $126.7(6)$ \\
\hline $\mathrm{P}(2)-\mathrm{Lu}(1)-\mathrm{Lu}(2)$ & $40.97(3)$ & $\mathrm{C}\left(22^{\prime}\right)-\mathrm{P}(3)-\mathrm{Lu}(1)$ & $131.4(6)$ \\
\hline $\mathrm{P}(3)-\mathrm{Lu}(1)-\mathrm{Lu}(2)$ & $110.49(4)$ & $\mathrm{C}(36)-\mathrm{P}(3)-\mathrm{Lu}(1)$ & $94.66(17)$ \\
\hline $\mathrm{P}(4)-\mathrm{Lu}(1)-\mathrm{Lu}(2)$ & $112.58(3)$ & $\mathrm{C}\left(19^{\prime}\right)-\mathrm{P}(3)-\mathrm{Lu}(1)$ & $135.7(8)$ \\
\hline $\mathrm{N}(2)-\mathrm{Lu}(2)-\mathrm{P}(2)$ & $125.49(10)$ & $\mathrm{C}(19)-\mathrm{P}(3)-\mathrm{Lu}(1)$ & $113.5(5)$ \\
\hline $\mathrm{N}(2)-\mathrm{Lu}(2)-\mathrm{P}(1)$ & $149.83(10)$ & $\mathrm{C}(22)-\mathrm{P}(3)-\mathrm{Lu}(1)$ & $112.1(4)$ \\
\hline $\mathrm{P}(2)-\mathrm{Lu}(2)-\mathrm{P}(1)$ & $83.39(4)$ & $\mathrm{C}(43)-\mathrm{P}(4)-\mathrm{C}(25)$ & $105.6(2)$ \\
\hline $\mathrm{N}(2)-\mathrm{Lu}(2)-\mathrm{P}(6)$ & $69.20(10)$ & $\mathrm{C}(43)-\mathrm{P}(4)-\mathrm{C}(28)$ & $104.4(2)$ \\
\hline $\mathrm{P}(2)-\mathrm{Lu}(2)-\mathrm{P}(6)$ & $109.48(4)$ & $C(25)-P(4)-C(28)$ & $104.4(3)$ \\
\hline $\mathrm{P}(1)-\mathrm{Lu}(2)-\mathrm{P}(6)$ & $94.67(5)$ & $\mathrm{C}(43)-\mathrm{P}(4)-\mathrm{Lu}(1)$ & $97.33(17)$ \\
\hline $\mathrm{N}(2)-\mathrm{Lu}(2)-\mathrm{P}(5)$ & $68.24(10)$ & $\mathrm{C}(25)-\mathrm{P}(4)-\mathrm{Lu}(1)$ & $123.00(19)$ \\
\hline $\mathrm{P}(2)-\mathrm{Lu}(2)-\mathrm{P}(5)$ & $109.42(4)$ & $\mathrm{C}(28)-\mathrm{P}(4)-\mathrm{Lu}(1)$ & $119.33(17)$ \\
\hline $\mathrm{P}(1)-\mathrm{Lu}(2)-\mathrm{P}(5)$ & $113.75(5)$ & $\mathrm{C}(62)-\mathrm{P}(5)-\mathrm{C}(54)$ & $106.4(3)$ \\
\hline$P(6)-L u(2)-P(5)$ & $133.75(4)$ & $\mathrm{C}(62)-\mathrm{P}(5)-\mathrm{C}(51)$ & $104.6(2)$ \\
\hline$N(2)-\operatorname{Lu}(2)-\operatorname{Lu}(1)$ & $166.87(10)$ & $\mathrm{C}(54)-\mathrm{P}(5)-\mathrm{C}(51)$ & $103.5(3)$ \\
\hline$P(2)-\operatorname{Lu}(2)-\operatorname{Lu}(1)$ & $42.42(3)$ & $\mathrm{C}(62)-\mathrm{P}(5)-\mathrm{Lu}(2)$ & $92.25(17)$ \\
\hline $\mathrm{P}(1)-\mathrm{Lu}(2)-\mathrm{Lu}(1)$ & $41.04(3)$ & $\mathrm{C}(54)-\mathrm{P}(5)-\mathrm{Lu}(2)$ & $122.4(2)$ \\
\hline$P(6)-\operatorname{Lu}(2)-\operatorname{Lu}(1)$ & $107.75(3)$ & $\mathrm{C}(51)-\mathrm{P}(5)-\mathrm{Lu}(2)$ & $123.94(17)$ \\
\hline$P(5)-\operatorname{Lu}(2)-\operatorname{Lu}(1)$ & 117.74(3) & $\mathrm{C}(69)-\mathrm{P}(6)-\mathrm{C}(45)$ & $104.9(2)$ \\
\hline $\mathrm{C}(1)-\mathrm{P}(1)-\mathrm{Lu}(1)$ & $135.85(18)$ & $\mathrm{C}(69)-\mathrm{P}(6)-\mathrm{C}(48)$ & $102.3(2)$ \\
\hline $\mathrm{C}(1)-\mathrm{P}(1)-\mathrm{Lu}(2)$ & $126.11(18)$ & $\mathrm{C}(45)-\mathrm{P}(6)-\mathrm{C}(48)$ & $104.9(2)$ \\
\hline $\mathrm{Lu}(1)-\mathrm{P}(1)-\mathrm{Lu}(2)$ & $96.97(5)$ & $\mathrm{C}(69)-\mathrm{P}(6)-\mathrm{Lu}(2)$ & $99.27(16)$ \\
\hline $\mathrm{C}(10)-\mathrm{P}(2)-\mathrm{Lu}(2)$ & $141.46(18)$ & $\mathrm{C}(45)-\mathrm{P}(6)-\mathrm{Lu}(2)$ & $126.76(16)$ \\
\hline
\end{tabular}




\begin{tabular}{|c|c|}
\hline $\mathrm{C}(48)-\mathrm{P}(6)-\mathrm{Lu}(2)$ & $115.22(16)$ \\
\hline $\mathrm{C}(31)-\mathrm{N}(1)-\mathrm{C}(38)$ & $116.6(4)$ \\
\hline $\mathrm{C}(31)-\mathrm{N}(1)-\mathrm{Lu}(1)$ & $120.5(3)$ \\
\hline $\mathrm{C}(38)-\mathrm{N}(1)-\mathrm{Lu}(1)$ & $122.8(3)$ \\
\hline $\mathrm{C}(64)-\mathrm{N}(2)-\mathrm{C}(57)$ & $116.6(4)$ \\
\hline $\mathrm{C}(64)-\mathrm{N}(2)-\mathrm{Lu}(2)$ & $127.5(3)$ \\
\hline $\mathrm{C}(57)-\mathrm{N}(2)-\mathrm{Lu}(2)$ & $115.9(3)$ \\
\hline$C(2)-C(1)-C(6)$ & $117.2(5)$ \\
\hline $\mathrm{C}(2)-\mathrm{C}(1)-\mathrm{P}(1)$ & $120.8(4)$ \\
\hline $\mathrm{C}(6)-\mathrm{C}(1)-\mathrm{P}(1)$ & $121.9(4)$ \\
\hline$C(3)-C(2)-C(1)$ & $119.5(5)$ \\
\hline$C(3)-C(2)-C(7)$ & $120.0(5)$ \\
\hline$C(1)-C(2)-C(7)$ & $120.4(4)$ \\
\hline$C(4)-C(3)-C(2)$ & $123.2(5)$ \\
\hline$C(5)-C(4)-C(3)$ & $116.8(5)$ \\
\hline$C(5)-C(4)-C(8)$ & $122.0(6)$ \\
\hline $\mathrm{C}(3)-\mathrm{C}(4)-\mathrm{C}(8)$ & 121.2(6) \\
\hline$C(4)-C(5)-C(6)$ & $123.3(6)$ \\
\hline$C(5)-C(6)-C(1)$ & $120.0(5)$ \\
\hline$C(5)-C(6)-C(9)$ & $120.9(5)$ \\
\hline $\mathrm{C}(1)-\mathrm{C}(6)-\mathrm{C}(9)$ & $119.1(5)$ \\
\hline $\mathrm{C}(11)-\mathrm{C}(10)-\mathrm{C}(15)$ & $117.3(4)$ \\
\hline $\mathrm{C}(11)-\mathrm{C}(10)-\mathrm{P}(2)$ & $120.4(4)$ \\
\hline $\mathrm{C}(15)-\mathrm{C}(10)-\mathrm{P}(2)$ & $122.3(4)$ \\
\hline$C(12)-C(11)-C(10)$ & $121.6(5)$ \\
\hline $\mathrm{C}(12)-\mathrm{C}(11)-\mathrm{C}(16)$ & $118.5(5)$ \\
\hline$C(10)-C(11)-C(16)$ & $119.9(5)$ \\
\hline$C(11)-C(12)-C(13)$ & $120.7(5)$ \\
\hline$C(14)-C(13)-C(12)$ & $118.1(5)$ \\
\hline$C(14)-C(13)-C(17)$ & $121.0(5)$ \\
\hline$C(12)-C(13)-C(17)$ & $120.9(5)$ \\
\hline$C(15)-C(14)-C(13)$ & $123.1(5)$ \\
\hline$C(14)-C(15)-C(10)$ & $119.2(5)$ \\
\hline$C(14)-C(15)-C(18)$ & $119.5(5)$ \\
\hline$C(10)-C(15)-C(18)$ & $121.3(4)$ \\
\hline $\mathrm{C}(20)-\mathrm{C}(19)-\mathrm{C}(21)$ & $109.8(15)$ \\
\hline
\end{tabular}

\begin{tabular}{|c|c|}
\hline$C(20)-C(19)-P(3)$ & $119.1(12)$ \\
\hline $\mathrm{C}(21)-\mathrm{C}(19)-\mathrm{P}(3)$ & $117.2(13)$ \\
\hline $\mathrm{C}\left(20^{\prime}\right)-\mathrm{C}\left(19^{\prime}\right)-\mathrm{C}\left(21^{\prime}\right)$ & $130(3)$ \\
\hline $\mathrm{C}\left(20^{\prime}\right)-\mathrm{C}\left(19^{\prime}\right)-\mathrm{P}(3)$ & $96.5(14)$ \\
\hline $\mathrm{C}\left(21^{\prime}\right)-\mathrm{C}\left(19^{\prime}\right)-\mathrm{P}(3)$ & $113.3(16)$ \\
\hline $\mathrm{C}(24)-\mathrm{C}(22)-\mathrm{C}(23)$ & $110.5(13)$ \\
\hline $\mathrm{C}(24)-\mathrm{C}(22)-\mathrm{P}(3)$ & $115.9(15)$ \\
\hline $\mathrm{C}(23)-\mathrm{C}(22)-\mathrm{P}(3)$ & $115.6(8)$ \\
\hline $\mathrm{C}\left(23^{\prime}\right)-\mathrm{C}\left(22^{\prime}\right)-\mathrm{C}\left(24^{\prime}\right)$ & $117.3(16)$ \\
\hline $\mathrm{C}\left(23^{\prime}\right)-\mathrm{C}\left(22^{\prime}\right)-\mathrm{P}(3)$ & $106.5(12)$ \\
\hline $\mathrm{C}\left(24^{\prime}\right)-\mathrm{C}\left(22^{\prime}\right)-\mathrm{P}(3)$ & $111.7(10)$ \\
\hline $\mathrm{C}(27)-\mathrm{C}(25)-\mathrm{C}(26)$ & $109.6(5)$ \\
\hline $\mathrm{C}(27)-\mathrm{C}(25)-\mathrm{P}(4)$ & $107.9(4)$ \\
\hline $\mathrm{C}(26)-\mathrm{C}(25)-\mathrm{P}(4)$ & $112.1(4)$ \\
\hline $\mathrm{C}(29)-\mathrm{C}(28)-\mathrm{C}(30)$ & $109.8(5)$ \\
\hline $\mathrm{C}(29)-\mathrm{C}(28)-\mathrm{P}(4)$ & $114.9(4)$ \\
\hline $\mathrm{C}(30)-\mathrm{C}(28)-\mathrm{P}(4)$ & $110.0(4)$ \\
\hline$C(36)-C(31)-C(32)$ & $116.8(5)$ \\
\hline $\mathrm{C}(36)-\mathrm{C}(31)-\mathrm{N}(1)$ & $120.3(4)$ \\
\hline $\mathrm{C}(32)-\mathrm{C}(31)-\mathrm{N}(1)$ & $122.8(5)$ \\
\hline $\mathrm{C}(33)-\mathrm{C}(32)-\mathrm{C}(31)$ & $122.0(5)$ \\
\hline $\mathrm{C}(32)-\mathrm{C}(33)-\mathrm{C}(34)$ & $121.5(5)$ \\
\hline$C(35)-C(34)-C(33)$ & $116.9(5)$ \\
\hline$C(35)-C(34)-C(37)$ & $121.5(5)$ \\
\hline $\mathrm{C}(33)-\mathrm{C}(34)-\mathrm{C}(37)$ & $121.6(5)$ \\
\hline $\mathrm{C}(34)-\mathrm{C}(35)-\mathrm{C}(36)$ & $122.3(5)$ \\
\hline $\mathrm{C}(31)-\mathrm{C}(36)-\mathrm{C}(35)$ & $120.5(5)$ \\
\hline $\mathrm{C}(31)-\mathrm{C}(36)-\mathrm{P}(3)$ & $115.2(4)$ \\
\hline $\mathrm{C}(35)-\mathrm{C}(36)-\mathrm{P}(3)$ & $124.2(4)$ \\
\hline $\mathrm{C}(43)-\mathrm{C}(38)-\mathrm{C}(39)$ & $117.1(5)$ \\
\hline $\mathrm{C}(43)-\mathrm{C}(38)-\mathrm{N}(1)$ & $120.8(4)$ \\
\hline $\mathrm{C}(39)-\mathrm{C}(38)-\mathrm{N}(1)$ & $122.0(5)$ \\
\hline $\mathrm{C}(40)-\mathrm{C}(39)-\mathrm{C}(38)$ & $121.1(5)$ \\
\hline$C(39)-C(40)-C(41)$ & $122.5(5)$ \\
\hline $\mathrm{C}(42)-\mathrm{C}(41)-\mathrm{C}(40)$ & $116.1(5)$ \\
\hline $\mathrm{C}(42)-\mathrm{C}(41)-\mathrm{C}(44)$ & $123.0(5)$ \\
\hline
\end{tabular}




\begin{tabular}{|c|c|c|c|}
\hline$C(40)-C(41)-C(44)$ & $120.8(5)$ & $\mathrm{C}(66)-\mathrm{C}(67)-\mathrm{C}(70)$ & $121.0(5)$ \\
\hline$C(41)-C(42)-C(43)$ & $123.2(5)$ & $C(67)-C(68)-C(69)$ & $122.7(5)$ \\
\hline $\mathrm{C}(42)-\mathrm{C}(43)-\mathrm{C}(38)$ & $119.9(5)$ & $\mathrm{C}(68)-\mathrm{C}(69)-\mathrm{C}(64)$ & $119.9(4)$ \\
\hline $\mathrm{C}(42)-\mathrm{C}(43)-\mathrm{P}(4)$ & $124.4(4)$ & $\mathrm{C}(68)-\mathrm{C}(69)-\mathrm{P}(6)$ & $124.0(4)$ \\
\hline $\mathrm{C}(38)-\mathrm{C}(43)-\mathrm{P}(4)$ & $115.7(4)$ & $\mathrm{C}(64)-\mathrm{C}(69)-\mathrm{P}(6)$ & $115.9(4)$ \\
\hline$C(46)-C(45)-C(47)$ & $111.0(4)$ & & \\
\hline $\mathrm{C}(46)-\mathrm{C}(45)-\mathrm{P}(6)$ & $110.6(4)$ & & \\
\hline $\mathrm{C}(47)-\mathrm{C}(45)-\mathrm{P}(6)$ & $111.3(3)$ & & \\
\hline$C(49)-C(48)-C(50)$ & $110.2(4)$ & & \\
\hline $\mathrm{C}(49)-\mathrm{C}(48)-\mathrm{P}(6)$ & $115.2(3)$ & & \\
\hline $\mathrm{C}(50)-\mathrm{C}(48)-\mathrm{P}(6)$ & $109.2(3)$ & & \\
\hline $\mathrm{C}(52)-\mathrm{C}(51)-\mathrm{C}(53)$ & $109.7(5)$ & & \\
\hline $\mathrm{C}(52)-\mathrm{C}(51)-\mathrm{P}(5)$ & 111.1(4) & & \\
\hline $\mathrm{C}(53)-\mathrm{C}(51)-\mathrm{P}(5)$ & $113.5(4)$ & & \\
\hline$C(56)-C(54)-C(55)$ & $111.5(5)$ & & \\
\hline $\mathrm{C}(56)-\mathrm{C}(54)-\mathrm{P}(5)$ & $107.9(4)$ & & \\
\hline $\mathrm{C}(55)-\mathrm{C}(54)-\mathrm{P}(5)$ & $110.4(4)$ & & \\
\hline$C(58)-C(57)-C(62)$ & $117.0(5)$ & & \\
\hline $\mathrm{C}(58)-\mathrm{C}(57)-\mathrm{N}(2)$ & $123.0(5)$ & & \\
\hline $\mathrm{C}(62)-\mathrm{C}(57)-\mathrm{N}(2)$ & $119.9(4)$ & & \\
\hline$C(59)-C(58)-C(57)$ & $121.8(5)$ & & \\
\hline$C(58)-C(59)-C(60)$ & $121.4(5)$ & & \\
\hline$C(61)-C(60)-C(59)$ & $117.5(5)$ & & \\
\hline$C(61)-C(60)-C(63)$ & $121.9(6)$ & & \\
\hline$C(59)-C(60)-C(63)$ & $120.6(5)$ & & \\
\hline$C(60)-C(61)-C(62)$ & $122.8(5)$ & & \\
\hline$C(61)-C(62)-C(57)$ & $119.5(5)$ & & \\
\hline $\mathrm{C}(61)-\mathrm{C}(62)-\mathrm{P}(5)$ & $124.7(4)$ & & \\
\hline $\mathrm{C}(57)-\mathrm{C}(62)-\mathrm{P}(5)$ & $115.8(4)$ & & \\
\hline $\mathrm{N}(2)-\mathrm{C}(64)-\mathrm{C}(65)$ & $123.4(4)$ & & \\
\hline $\mathrm{N}(2)-\mathrm{C}(64)-\mathrm{C}(69)$ & $119.7(4)$ & & \\
\hline$C(65)-C(64)-C(69)$ & $116.8(4)$ & & \\
\hline$C(66)-C(65)-C(64)$ & $121.6(5)$ & & \\
\hline$C(65)-C(66)-C(67)$ & $121.8(5)$ & & \\
\hline$C(68)-C(67)-C(66)$ & $117.1(5)$ & & \\
\hline$C(68)-C(67)-C(70)$ & $121.8(5)$ & & \\
\hline
\end{tabular}




\section{References}

1. Fan, L.; Foxman, B. M.; Ozerov, O. V. J. Am. Chem. Soc. 2004, 23, 326-328.

2. Arndt, S.; Voth, P.; Spaniol, T. P.; Okuda, J. Organometallics 2000, 19, 4690-4700.

3. Becker, G.; Mundt, O.; Rössler, M.; Schneider, E. Z. Anorg. Allg. Chem. 1978, 443, 42-52.

4. Liddle, S. T.; Izod, K. Organometallics, 2004, 23, 5550-5559.

5. Fan, L. Ph.D. Thesis, Brandeis University, 2006.

6. Brym, M.; Jones, C.; Waugh, M; Hey-Hawkins, E.; Majoumo, F. New J. Chem. 2003, 27, 1614-1621.

7. Klebach, Th. C.; Lourens, R.; Bickelhaupt, F. J. Am. Chem. Soc. 1978, 100, 4886-4888.

8. Böhm, V. P. W.; Brookhart, M. Angew. Chem. Int. Ed. 2001, 40, 4694-4696.

9. $\quad$ APEX II 1.08; Bruker AXS, Inc.: Madison, WI 53719, 2004.

10. SAINT+ 7.06; Bruker AXS, Inc.: Madison, WI 53719, 2003.

11. Sheldrick, G. SADABS 2.03; University of Göttingen, Germany, 2001.

12. SHELXTL Version 5.10; Bruker AXS, Inc.: Madison, WI 53719, 1997.

13. Farrugia, J. J. Appl. Cryst. 1997, 30, 565.

14. Spek, A. L. J. Appl. Cryst. 2003, 36, 7-13. 